This item was submitted to Loughborough's Research Repository by the author.

Items in Figshare are protected by copyright, with all rights reserved, unless otherwise indicated.

\title{
Superlight small bipolarons from realistic long-range Coulomb and Frohlich interactions
}

PLEASE CITE THE PUBLISHED VERSION

http://dx.doi.org/10.1103/PhysRevB.85.104520

PUBLISHER

(c) Americal Physical Society

VERSION

VoR (Version of Record)

LICENCE

CC BY-NC-ND 4.0

REPOSITORY RECORD

Alexandrov, A.S., J.H. Samson, and G. Sica. 2019. "Superlight Small Bipolarons from Realistic Long-range Coulomb and Frohlich Interactions”. figshare. https://hdl.handle.net/2134/11606. 
This item was submitted to Loughborough's Institutional Repository (https://dspace.lboro.ac.uk/) by the author and is made available under the following Creative Commons Licence conditions.

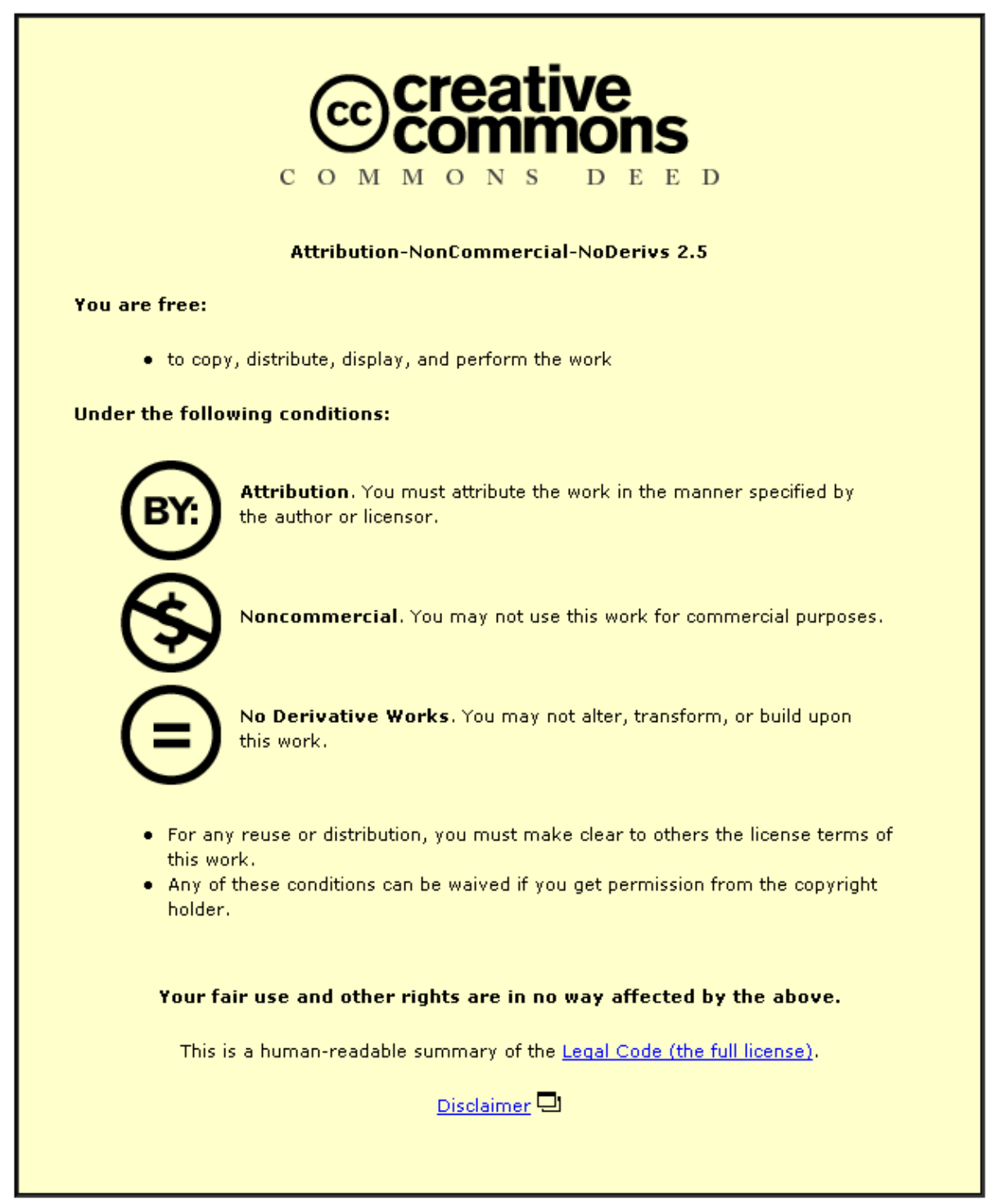

For the full text of this licence, please go to: http://creativecommons.org/licenses/by-nc-nd/2.5/ 


\title{
Superlight small bipolarons from realistic long-range Coulomb and Fröhlich interactions
}

\author{
A. S. Alexandrov, ${ }^{1,2}$ J. H. Samson, ${ }^{1}$ and G. Sica ${ }^{1,3}$ \\ ${ }^{1}$ Department of Physics, Loughborough University, Loughborough LE11 3TU, United Kingdom \\ ${ }^{2}$ Instituto de Fisica "Gleb Wataghin," Universidade Estadual de Campinas, UNICAMP 13083-970, Campinas, São Paulo, Brazil \\ ${ }^{3}$ Dipartimento di Fisica “E.R. Caianiello," Università degli Studi di Salerno, I-84084 Fisciano, Salerno, Italy
}

(Received 15 November 2011; revised manuscript received 16 March 2012; published 26 March 2012)

\begin{abstract}
We report analytical and numerical results on the two-particle states of the polaronic $t$ - $J_{p}$ model derived recently with realistic Coulomb and electron-phonon (Fröhlich) interactions in doped polar insulators. Eigenstates and eigenvalues are calculated for two different geometries. Our results show that the ground state is a bipolaronic singlet, made up of two polarons. The bipolaron size increases with increasing ratio of the polaron hopping integral $t$ to the exchange interaction $J_{p}$ but remains small in the whole range $0 \leqslant t / J_{p} \leqslant 1$. Furthermore, the model exhibits a phase transition to a superconducting state with a critical temperature well in excess of $100 \mathrm{~K}$ since the small bipolarons are perfectly mobile. In the range $t / J_{p} \leqslant 1$, there are distinct charge and spin gaps opening in the density of states, specific heat, and magnetic susceptibility well above $T_{c}$.
\end{abstract}

DOI: 10.1103/PhysRevB.85.104520

PACS number(s): 71.38.-k

With few exceptions, ${ }^{1}$ it is widely believed that the conventional Bardeen-Cooper-Schrieffer (BCS) theory and its intermediate-coupling Eliashberg extension ${ }^{2}$ do not suffice to explain high-temperature superconductivity. On the contrary, there is growing understanding that the true origin of hightemperature superconductivity should be found in a proper combination of the Coulomb repulsion with a significant electron-phonon interaction (EPI). ${ }^{3}$ The many-body theory of strongly correlated electrons and phonons was originally developed with the on-site Hubbard repulsion and the short-range Holstein EPI using analytical strong-coupling expansion ${ }^{4}$ and powerful numerical techniques ${ }^{5}$ in the framework of the Hubbard-Holstein and Holstein- $t-J$ models. ${ }^{6}$ Also the manybody Coulomb-Fröhlich model, which takes into account a finite range of realistic interactions, was proposed ${ }^{7}$ and studied analytically ${ }^{8}$ and numerically, ${ }^{9}$ showing a rich phase diagram with a polaronic Fermi-liquid, superconductivity induced by mobile bipolarons and a charge-segregated phase. In these and many other studies ${ }^{10}$ both interactions were introduced as input parameters not directly related to the material.

Recently it has been shown that, in highly polarizable ionic lattices, the bare long-range Coulomb and electron-phonon interactions almost negate each other, giving rise to a physics described by the polaronic $t-J_{p}$ model $^{11}$ with a short-range polaronic spin-exchange $J_{p}$ of phononic origin,

$$
\begin{aligned}
\mathcal{H} \equiv & -\sum_{i, j} t_{i j} \delta_{\sigma \sigma^{\prime}} c_{i}^{\dagger} c_{j}+2 \sum_{\mathbf{m} \neq \mathbf{n}} J_{p}(\mathbf{m}-\mathbf{n}) \\
& \times\left(\mathbf{S}_{\mathbf{m}} \cdot \mathbf{S}_{\mathbf{n}}+\frac{1}{4} n_{\mathbf{m}} n_{\mathbf{n}}\right)
\end{aligned}
$$

Here the sum over $\mathbf{n} \neq \mathbf{m}$ counts each pair once only, $\mathbf{S}_{\mathbf{m}}=(1 / 2) \sum_{\sigma, \sigma^{\prime}} c_{\mathbf{m} \sigma}^{\dagger} \vec{\tau}_{\sigma \sigma^{\prime}} c_{\mathbf{m} \sigma^{\prime}}$ is the spin $\frac{1}{2}$ operator $(\vec{\tau}$ are the Pauli matrices), $n_{\mathbf{m}}=\sum_{\sigma} c_{i}^{\dagger} c_{i}$, and $i=(\mathbf{m}, \sigma)$ and $j=\left(\mathbf{n}, \sigma^{\prime}\right)$ include both site $(\mathbf{m}, \mathbf{n})$ and $\operatorname{spin}\left(\sigma, \sigma^{\prime}\right)$ indices; $t_{i j}$ is the polaron hopping integral while $J_{p}(\mathbf{m}-\mathbf{n})>t$ represents the exchange interaction between polarons on different sites from a residual polaron-multiphonon interaction. It has been proposed that the $t-J_{p}$ Hamiltonian, Eq. (1), has a high- $T_{c}$ superconducting ground state protected from clustering. ${ }^{11}$
In this work we present numerical and analytical results on the two-particle eigenstates of the polaronic $t-J_{p}$ model as the building blocks for high-temperature superconductivity. It is worth noting that there is a wide difference between (1) and the familiar $t-J$ model $^{12}$ derived from the repulsive Hubbard $U$ Hamiltonian in the limit $U \gg t$ omitting the so-called three-site hoppings and EPI. The latter model acts in a projected Hilbert space constrained to no double occupancy. On the contrary $t-J_{p}$ Hamiltonian, Eq. (1) has no constraint on the on-site occupancy since the on-site Coulomb repulsion is negated by the Fröhlich EPI. The hopping integral $t_{i j}$ leads to the coherent (bi)polaron band while the antiferromagnetic exchange $J_{p}$ bounds polarons into superlight intersite bipolarons. Moreover, the sign "+" instead of "-" in the last density-density interaction term in (1) provides an effective repulsion between pairs, preventing their clustering, ${ }^{13}$ while the repulsive $t-J$ model favors a phase separation.

Also different from any model proposed so far is that all quantities in the polaronic $t-J_{p}$ Hamiltonian (1) are defined through the material parameters, in particular $t_{i j}=T(\mathbf{m}-$ n) $\exp \left[-g^{2}(\mathbf{m}-\mathbf{n})\right]$ with

$$
g^{2}(\mathbf{m})=\frac{2 \pi e^{2}}{\kappa \hbar \omega_{0} V} \sum_{\mathbf{q}} \frac{1-\cos (\mathbf{q} \cdot \mathbf{m})}{q^{2}}
$$

and

$$
J_{p}(\mathbf{m})=T^{2}(\mathbf{m}) / 2 g^{2}(\mathbf{m}) \hbar \omega_{0},
$$

where $\kappa=\epsilon_{\infty} \epsilon_{0} /\left(\epsilon_{0}-\epsilon_{\infty}\right)$ and $V$ is the normalization volume. Here the high-frequency $\epsilon_{\infty}$ and the static $\epsilon_{0}$ dielectric constants as well as the optical phonon frequency $\omega_{0}$ and the bare hopping integrals in a rigid lattice $T(\mathbf{m})$ are measured and/or found using first-principle density functional theory ${ }^{14}$ in a parent polar insulator. Our $J_{p}$ describes the spin exchange of carriers doped into polar insulators as, for instance, oxygen holes in the cuprates, rather than antiferromagnetic correlations of copper spins in the parent insulator, which are well described by the conventional $J$. The polaronic $J_{p}$ (on the order of a few hundred meV or more) is larger than $J \approx 100 \mathrm{meV}$. 
(a)

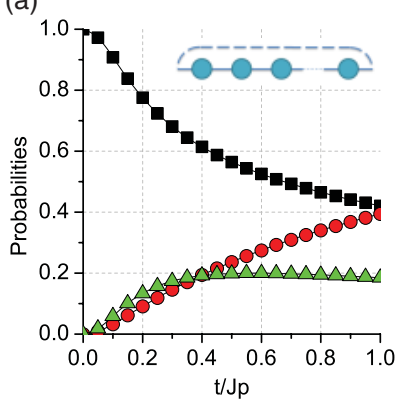

(b)

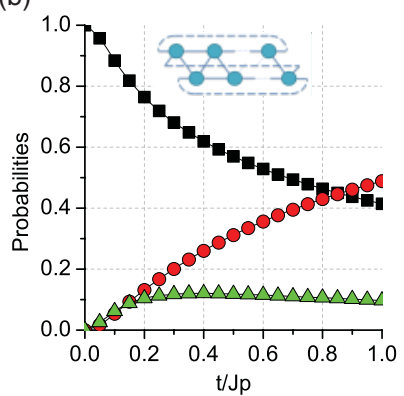

FIG. 1. (Color online) Probability of finding two polarons on the nearest-neighbor sites: $P_{b p}$ (squares), on more distant sites (circles) and on the same site (triangles) in the ground state of the $t-J_{p}$ Hamiltonian for chain (a) and zigzag ladder (b).

In the following we restrict the range of the exchange interaction and the hopping to nearest neighbors. One can readily find ${ }^{13}$ highly degenerate two-particle energy levels of the model (1) for $t=0$,

$E_{0}(t=0)=-J_{p}, \quad E_{1}(t=0)=0, \quad E_{2}(t=0)=J_{p}$.

The ground and the highest energy states are bipolaronic spin-singlet and spin-triplet, respectively, made up of two polarons on neighboring sites. The zero-energy states are combinations of pairs of polarons separated by more than one lattice parameter and on-site bipolarons, since there are no on-site interaction terms in the Hamiltonian (1).

For $t \neq 0$ there is a finite bandwidth associated with each of the three energy levels. Exact diagonalization (ED) results show that the ground-state configuration is virtually unchanged since the distance between two bound polarons remains of the order of the lattice spacing as long as $t<J_{p}$. In that range in fact, regardless of the particular geometry, the probability $P_{b p}$ to find two polarons on nearest-neighbor sites decreases gradually with increasing $t / J_{p}$ and remains finite as shown in Fig. 1.

We also show in Fig. 2 the ground-state energy as a function of the $t / J_{p}$ ratio for each analyzed geometry. Importantly, fitting the ED results in the $t \ll J_{p}$ range, there is a contribution linear in $t$ in the zigzag ladder where a single hopping

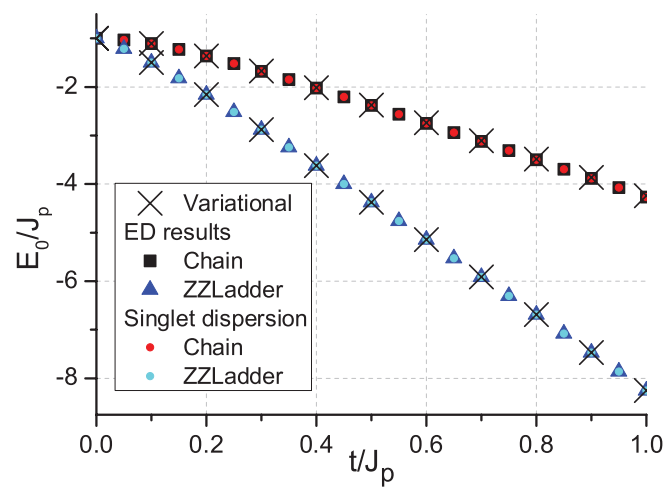

FIG. 2. (Color online) Two-particle ground-state energy $E_{0}$ as a function of the hopping. Symbols correspond to ED (squares and triangles) and variational (crosses) data on finite clusters. We also report (circles) the results at $k=0$ obtained by diagonalizing $\hat{H}(k)$ given in Eq. (6).

is sufficient for the coherent propagation of the intersite bipolaron through the lattice. ${ }^{7}$ On the contrary, in the case of a one-dimensional chain the bipolaron hopping is realized through a second-order process, resulting in the quadratic behavior of the ground-state energy as in the case of the on-site bipolaron. ${ }^{4}$ As shown in Fig. 2, ED results are in excellent agreement with the ones that can be obtained by means of the variational method developed by Bonča et al. ${ }^{15}$

Beyond ED results, additional information on the twoparticle dynamics can be obtained by considering the following two-particle singlet basis for an infinite lattice:

$|\mathbf{m}, \mathbf{k}\rangle$

$$
=\left\{\begin{array}{ll}
\frac{1}{\sqrt{2 N}} \sum_{\mathbf{n}} e^{i \mathbf{k} \cdot\left(\mathbf{n}+\frac{\mathbf{m}}{2}\right)}\left(c_{\mathbf{n} \uparrow}^{\dagger} c_{\mathbf{n}+\mathbf{m} \downarrow}^{\dagger}+c_{\mathbf{n}+\mathbf{m} \uparrow}^{\dagger} c_{\mathbf{n} \downarrow}^{\dagger}\right)|0\rangle, & m>0 \\
\frac{1}{\sqrt{N}} \sum_{\mathbf{n}} e^{i \mathbf{k} \cdot \mathbf{n}} c_{\mathbf{n} \uparrow}^{\dagger} c_{\mathbf{n} \downarrow}^{\dagger}|0\rangle, & m=0
\end{array} .\right.
$$

For the analyzed one-dimensional geometries the matrix representation of the $t-J_{p}$ Hamiltonian in this basis is

$$
\hat{H}(k)=\left(\begin{array}{ccccc}
0 & \sqrt{2} e_{1}(k) & \sqrt{2} e_{2}(k) & 0 & \ldots \\
\sqrt{2} e_{1}(k) & -J_{p}+e_{2}(k) & e_{1}(k) & e_{2}(k) & \ddots \\
\sqrt{2} e_{2}(k) & e_{1}(k) & e_{3} & e_{1}(k) & \ddots \\
0 & e_{2}(k) & e_{1}(k) & 0 & \ddots \\
\vdots & \ddots & \ddots & \ddots & \ddots
\end{array}\right),
$$

where $e_{1}(k)=-2 t \cos (k a / 2)$ and $e_{2}(k)=e_{3}=0$ for the chain while we have $e_{1}(k)=-2 t \cos (k a / 4), e_{2}(k)=$ $-2 t \cos (k a / 2)$, and $e_{3}=-J_{p}$ for the zigzag ladder.

The eigenvalues of the tridiagonal matrix $\hat{H}(k)$ determine the energy dispersion $E(k)$. In the limit $t \rightarrow 0, e_{i}(k) \rightarrow 0$, so that the ground-state energy is $-J_{p}$, in agreement with the ED results showed so far. For any $t>0$, the problem is still solvable by requiring the system wave function to decay exponentially in the region where the potential vanishes. ${ }^{13}$ In the case of a chain, the energy dispersion can be derived from a cubic equation that, in the $t \ll J_{p}$ limit, gives

$$
E_{s}(k)=-J_{p}-\left(12 t^{2} / J_{p}\right) \cos ^{2}(k a / 2)+O\left(t^{4}\right)
$$

with a quadratic contribution with respect to the hopping term. On the contrary, in the same limit the corresponding dispersion for the zigzag ladder ${ }^{16}$ has been found to be linear in $t$ :

$$
E_{s}(k)=-J_{p}-t\left[\cos (k a / 2)+\sqrt{1+4 \cos ^{4}(k a / 4)}\right]+O\left(t^{2}\right) .
$$

As shown in Fig. 2, the energy dispersions at $k=0$ obtained for the chain and the zigzag ladder are in perfect agreement with ED and variational results on finite clusters in the whole range $0 \leqslant t / J_{p}<1$.

These results allow for some insight into a possible superconducting phase transition and pseudogap signatures in the response functions of the model.

According to the Mermin-Wagner theorem, ${ }^{17}$ there should be no phase transition at finite temperatures in one and two 
dimensions (1D and 2D) since there is no continuous symmetry breaking. However, a finite temperature phase transition in 2D can exist via the Berezinsky-Kosterlitz-Thouless (BKT) mechanism. ${ }^{18,19}$ For a hard-core 2D Bose gas, where the BoseEinstein condensation does not occur, ${ }^{20,21}$ a phase transition to a superfluid state is expected. ${ }^{19}$ In particular, it has been shown 22 in the dilute limit $\ln \ln \left(1 / n_{b} r^{2}\right)>1$ that the critical temperature is

$$
T_{c}=2 \pi \hbar^{2} n_{b} / k_{B} m^{* *} \ln \ln \left(1 / n_{b} r^{2}\right),
$$

where $n_{b}$ is the boson density per unit area and $r$ is the range of the boson-boson repulsion. In our case $r \approx a$ and $m^{* *}$ is the bipolaron mass.

To estimate the bipolaron effective mass for a 1D chain, one can use our dispersion (7), which gives $m^{* *}=\hbar^{2} J_{p} / 6 a^{2} t^{2}$. In the case of a zigzag ladder, taking into account only the linear contribution in $t / J_{p}$ results in an overestimated $m^{* *}$ with $m^{* *} / m^{*} \approx 5$ where $m^{*}=2 \hbar^{2} / 5 t a^{2}$ is the polaron mass. ${ }^{16}$ Numerical results obtained by solving the eigenvalue problem of (6) show in fact that the ratio $m^{* *} / m^{*}$ is below this estimate (Fig. 3). In particular, for $t \approx J_{p}$ we have $m^{* *} \approx 2 m^{*}$ for both chain and zigzag ladder geometries.

Since the effective mass is proportional to $1 / t^{2}$ or $1 / t$, one may conclude that $T_{c}$ should increase as $t$ or $t^{2}$ with the polaron hopping integral. On the other hand, our ED results, Fig. 1, show that the probability $P_{b p}\left(t / J_{p}\right)$ to find a hard-core tightly bound singlet decreases as the hopping increases. At sufficiently low density, BEC should not depend on whether the bipolarons are nearest neighbor or next nearest neighbor, so long as they are bound and the bipolaron spacing is much greater than the typical polaron separation. On the other hand when bipolarons overlap, their condensation appears in the form of the Cooper pairs in the momentum space with a lower critical temperature, rather than in real space (BEC-BCS crossover $\left.{ }^{4}\right)$. Hence, bounds for the critical temperature can be estimated by weighting Eq. (9) with $P=P_{b p}\left(t / J_{p}\right)$ as $T_{c}^{r} \approx P_{b p}\left(t / J_{p}\right) T_{c}$. As shown in Fig. 3, despite the low carrier density, the critical temperature is about $200 \mathrm{~K}$ with the chain and the zigzag ladder effective mass. In particular, $T_{c}^{r}$ obtained for the chain should not be considered as strictly related to the geometry but to the values of $m^{* *}$ that could be a crude (but
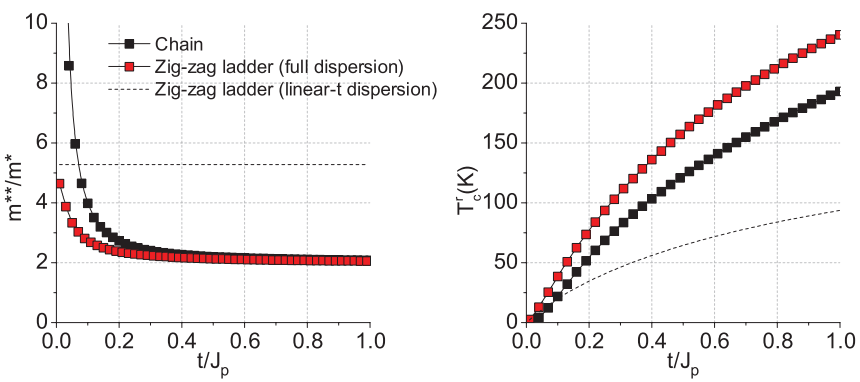

FIG. 3. (Color online) Ratio of bipolaron to polaron mass (left panel) in the $t-J_{p}$ model for different lattices and the resulting critical temperature (right panel) estimated with this mass at $n_{b}=$ $0.01 / a^{2}$ and $J_{p}=1.0 \mathrm{eV}$. For the zigzag ladder we report both the results obtained by using the linear- $t$ dispersion ${ }^{11}$ (dashed line) and the complete one (squares) calculated numerically from (6) by diagonalizing $\hat{H}(k)$ quite reliable) estimation of the bipolaron effective mass for a 2D lattice in the low-density limit. In the case of cuprate superconductors with the polaron binding energy in the range $0.5 \mathrm{eV} \leqslant E_{p} \leqslant 1.0 \mathrm{eV}$ and $0.3 \mathrm{eV} \leqslant J_{p} \leqslant 1.0 \mathrm{eV}$ (Ref. 11) one gets the realistic value of the bare hopping integral $0.2 \mathrm{eV} \leqslant T(a) \leqslant 0.4 \mathrm{eV}$ that gives $0.05 \leqslant t / J_{p} \leqslant 0.27$ and the critical temperature $20 \mathrm{~K} \leqslant T_{c} \leqslant 100 \mathrm{~K}$ at $n_{b}=0.01$.

Let us finally analyze the (pseudo)gap features in the density of states (DOS) and the spin susceptibility $\chi_{s}$ of the polaronic $t-J_{p}$ model at high temperatures well above $T_{c}^{r}$, when all carriers are nondegenerate. It is convenient to introduce the "occupation density of states" (ODOS), $\rho(\omega, T)$, by weighting the standard temperature-independent DOS with the Fermi-Dirac and the Bose-Einstein distribution functions,

$$
\rho(\omega, T) \equiv f_{s}(\omega, T) \mathcal{N}_{s}(\omega)+2 f_{p}(\omega, T) \mathcal{N}_{p}(\omega),
$$

where

$$
\mathcal{N}_{s, p}(\omega)=\frac{a}{2 \pi} \int_{-\pi / a}^{\pi / a} d k \delta\left[\omega-E_{s, p}(k)\right],
$$

and $f_{s, p}(\omega, T)=\left[\exp \left(\left(\omega-\mu_{s, p}\right) / k_{B} T\right) \mp 1\right]^{-1}$. Here $E_{s, p}(k)$ is the (bi)polaron dispersion and $\mu_{s}=2 \mu, \mu_{p}=\mu$ are the chemical potentials of bipolarons and single polarons, respectively, with $\mu<E_{s}(0) / 2$.

In the $t=0$ limit, according to (4) we have three different two-particle energy levels with $E_{s}(k)$ and $E_{p}(k)$ separated by $J_{p}$. However, at low carrier density and temperature, the highest energy level does not contribute to ODOS and we observe two sharp peaks at $\omega / J_{p}=-1$ and $\omega / J_{p}=0.0$ with a suppression of ODOS around $\omega / J_{p}=-0.5$. Hence there is a single charge/spin pseudogap, $\Delta_{c}=\Delta_{s}=J_{p}$. The ODOS for finite values of $t / J_{p}$ and temperatures is shown in Fig. 4 with some Gaussian broadening in the $\delta$ function in Eq. (11), modeling for instance disorder effects. At any $t \neq 0$, the two peaks become wider as $t / J_{p}$ increases and the gap between the bipolaron and the unpaired polaron bands gradually closes. With increasing temperature the single-particle polaron band is more populated along with the increasing population of higher energy levels in the bipolaron band, so that ODOS reflects a competition between bound and unbound states in the response functions. In particular, the behavior of the spin susceptibility indicates the presence of a finite spin-gap $\Delta_{s}$ that decreases gradually as $t$ becomes comparable with the binding energy
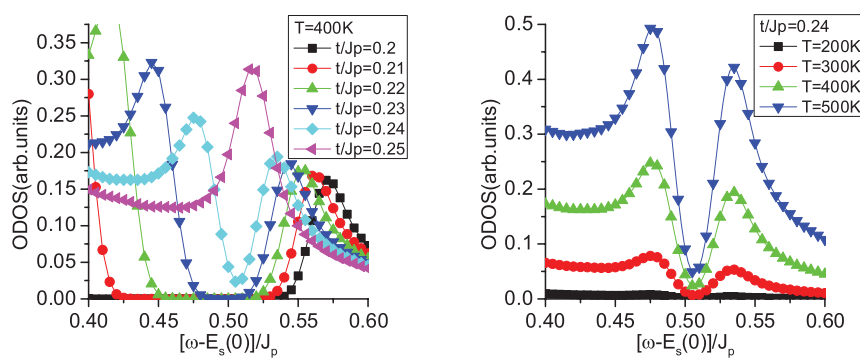

FIG. 4. (Color online) Signatures of a pseudogap opening in ODOS for different values of the polaron hopping (left panel) and temperature (right panel) calculated for the chain with a Gaussian broadening $\delta=0.01 J_{p}$, modeling a disorder effect in the $\delta$ function in Eq. (11). 
$J_{p},{ }^{13}$ coherently with the suppression of the probability to find a bipolaron in the nearest-neighbor configuration (Fig. 1). With increasing temperature, the number of occupied states within the pseudogap also increases. Without many-body correlation effects (i.e., a screening of the Coulomb and electron-phonon interactions at finite carrier densities ${ }^{23}$ ), the pseudogap itself does not depend on temperature; it is a matter of whether the temperature is high enough for single-polaron states to have significant occupation. Hence a characteristic pseudogap temperature $T^{*}$ exists in our model above which the pseudogap is suppressed, but it is a crossover temperature rather than a critical temperature. Further signatures of pseudogap opening are also found in the specific heat ${ }^{13}$ where the Schottky anomaly is induced by thermal excitation within the bipolaronic and the polaronic bands.

In conclusion, we have described some key features of the $t-J_{p}$ Hamiltonian in the low-density limit. We have shown that the ground-state configuration is a small bipolaron singlet. Depending on the competition between the hopping $t$ and the polaronic exchange interaction $J_{p}$, the bipolaron size changes but remains small in the whole range $0 \leqslant t / J_{p} \leqslant 1$. We have also argued that, in the $2 \mathrm{D}$ case, the presence of small light bipolarons results in a phase transition to a superconducting state at a critical temperature in excess of a hundred degrees Kelvin. Finally, the spin susceptibility and the specific heat of the model revealed charge and spin gaps. Because of the presence of a continuum spectrum, there is no true ground-state gap at any finite value of the polaron hopping $t$. However, strong evidence of a finite pseudogap has been found in the range where $t<J_{p}$ above $T_{c}$.

We gratefully acknowledge enlightening discussions with Annette Bussmann-Holder, Jorge Hirsch, Victor Kabanov, Hugo Keller, Ferdinando Mancini, Frank Marsiglio, and Roman Micnas and the support of the Royal Society (London), the UNICAMP visiting professorship program, and ROBOCON (Campinas, Brazil).
${ }^{1}$ E. G. Maksimov, M. L. Kulić, and O. V. Dolgov, Adv. Condens. Matter Phys. 2010, 423725 (2010).

${ }^{2}$ G. M. Eliashberg, Zh. Eksp. Teor. Fiz. 39, 1437 (1960) [Sov. Phys. JETP 12, 1000 (1960)].

${ }^{3}$ A. S. Alexandrov, C. Di Castro, I. Mazin, and D. Mihailovic, Adv. Condens. Matter Phys. 2010, 206012 (2010), and other contributions to this special issue.

${ }^{4}$ A. S. Alexandrov and J. Ranninger, Phys. Rev. B 23, 1796 (1981); A. S. Alexandrov, Russ. J. Phys. Chem. 57, 167 (1983).

${ }^{5}$ For reviewes see H. Fehske and S. A. Trugman, in Polarons in Advanced Materials, ed. A. S. Alexandrov (Springer, Dordrecht, the Netherlands, 2007), pp 393-461; A. S. Mishchenko and N. Nagaosa, ibid., pp. 503-544.

${ }^{6}$ L. Vidmar, J. Bonča, S. Maekawa, and T. Tohyama, Phys. Rev. Lett. 103, 186401 (2009).

${ }^{7}$ A. S. Alexandrov, Phys. Rev. B 53, 2863 (1996).

${ }^{8}$ A. S. Alexandrov and P. E. Kornilovitch, J. Phys.: Condens. Matter 14, 5337 (2002).

${ }^{9}$ T. M. Hardy, J. P. Hague, J. H. Samson, and A. S. Alexandrov, Phys. Rev. B 79, 212501 (2009); J. P. Hague, P. E. Kornilovitch, J. H. Samson, and A. S. Alexandrov, Phys. Rev. Lett. 98, 037002 (2007).

${ }^{10}$ For a recent review see A. S. Alexandrov and J. T. Devreese, Advances in Polaron Physics (Springer, Berlin, 2009).
${ }^{11}$ A. S. Alexandrov, Europhys. Lett. 95, 27004 (2011).

${ }^{12}$ J. E. Hirsch, Phys. Rev. Lett. 54, 1317 (1985); J. Spalek, Phys. Rev. B 37, 533 (1988); C. Gros, R. Joynt, and T. M. Rice, ibid. 36, 381 (1987).

${ }^{13}$ A. S. Alexandrov, J. H. Samson, and G. Sica (unpublished).

${ }^{14}$ T. Bauer and C. Falter, Phys. Rev. B 80, 094525 (2009).

${ }^{15}$ J. Bonča, S. A. Trugman, and I. Batistić, Phys. Rev. B 60, 1633 (1999); J. Bonča, T. Katrašnik, and S. A. Trugman, Phys. Rev. Lett. 84, 3153 (2000).

${ }^{16}$ The difference with respect to the one reported in Ref. [11] is due to an incorrect overall sign in the four bipolaron branches. The corrected dispersion gives $50-20 \sqrt{5} \approx 5$ for the bipolaronto-polaron mass ratio, instead of 10 as reported in Ref. [11].

${ }^{17}$ N. D. Mermin and H. Wagner, Phys. Rev. Lett. 17, 1133 (1966).

${ }^{18}$ V. L. Berezinskii, Zh. Eksp. Teor. Fiz. 61, 1144 (1971) [JETP Lett. 34, 610 (1972)].

${ }^{19}$ J. M. Kosterlitz and D. J. Thouless, J. Phys. C 6, 1181 (1973).

${ }^{20}$ J. D. Gunton and M. J. Buckingham, Phys. Rev. 166, 152 (1968).

${ }^{21}$ P. C. Hohenberg, Phys. Rev. 158, 383 (1967).

${ }^{22}$ D. S. Fisher and P. C. Hohenberg, Phys. Rev. B 37, 4936 (1988).

${ }^{23}$ A. S. Alexandrov and A. M. Bratkovsky, Phys. Rev. Lett. 105, 226408 (2010) 